\title{
A relação entre a geração de resíduos sólidos domiciliares e os estratos socioeconômicos
}

Este estudo se propõe a analisar a composição e a quantidade de resíduos sólidos domiciliares gerados em Juazeiro do Norte, Ceará, no nordeste brasileiro, relacionando-se a geração de resíduos com o estrato socioeconômico do bairro gerador. Para tanto, foram realizadas 727 coletas de resíduos domiciliares, gerados em seis bairros e em três períodos distintos no ano de 2016. Foram coletados $1.271,08 \mathrm{~kg}$ de resíduos. Houve uma predominância de resíduos orgânicos, atingindo uma média percentual de 58,51\%, seguida de materiais com potencial reciclável (com média de 17,02\%). A respeito dos materiais recicláveis, a maior geração fo identificada no bairro de estrato socioeconômico A2, em função do padrão de consumo de alimentos mais processados, indicando maior geração de resíduos de embalagens. A geração per capita diária de resíduos ( $\mathrm{kg} / \mathrm{habitante} / \mathrm{dia}$ ) resultou em uma média de $0,47 \mathrm{~kg} / \mathrm{hab} / \mathrm{dia}$. Observou-se que a geração per capita de resíduos sólidos domiciliares apresentou uma relação positiva de acordo com o nível de renda do bairro gerador, porém diminuiu quando atingiu o nível mais elevado de rendimentos, guardando semelhança empírica com a hipótese da Curva Ambiental de Kuznets. Salienta-se que a estratificação em perfis socioeconômicos é importante para investigar os padrões de geração de resíduos domésticos nos centros urbanos e contribui com informações mais assertivas para o gerenciamento dos resíduos sólidos.

Palavras-chave: Resíduos Sólidos Domiciliares; Análise Gravimétrica; Estrato Socioeconômico.

\section{The relationship between domestic solid waste generation and socioeconomic strata}

\begin{abstract}
This study aims to analyze the composition and quantity of domestic solid waste generated in Juazeiro do Norte, Ceará, in the Brazilian Northeast, relating the generation of waste with the socioeconomic stratum of the generating district. For that purpose, 727 door-to-door collections of household waste, generated in six districts of the city, were carried out in the periods: April 04 to 08 ; June 20-24; And, August 15 to 19, 2016. Waste was collected 1,271.08 kg. There was a predominance of organic residues, reaching a mean percentage of $58.51 \%$, followed by materials with recyclable potential (with an average of $17.02 \%$ ). Regarding recyclable materials, the largest generation was identified in the neighborhood of socioeconomic stratum 'A2', due to the pattern of consumption of more processed foods, indicating greater generation of packaging waste. The daily per capita generation of waste $(\mathrm{kg} / \mathrm{inh}$ habitant/day) resulted in an average of 0.47 $\mathrm{kg} / \mathrm{inh} a \mathrm{~b} /$ day. It was observed that the per capita generation of household solid waste presented a positive relation according to the income level of the generating district, but decreased when it reached the highest level of income, keeping empirical similarity to the hypothesis of the Kuznets Environmental Curve. It should be stressed that stratification in socioeconomic profiles is important to investigate the patterns of domestic waste generation in urban centers and contribute with more assertive information for solid waste management.
\end{abstract}

Keywords: Household Solid Waste; Gravimetric Analysis; Socioeconomic Status.

Topic: Valoração e Economia Ambiental

Reviewed anonymously in the process of blind peer.
Received: $10 / 04 / 2018$

Approved: 24/05/2018
Anny Kariny Feitosa (D)

Instituto Federal do Ceará, Brasil

http://lattes.cnpq.br/6604861578850716

http://orcid.org/0000-0002-5745-2660

akfeitosa@hotmail.com

Júlia Elisabete Barden (iD)

Universidade do Vale do Taquari, Brasil

http://lattes.cnpq.br/2618070777171361

http://orcid.org/0000-0002-9818-1844

ibarden@univates.br

\section{Odorico Konrad (iD)}

Universidade do Vale do Taquari, Brasil

http://lattes.cnpq.br/9946679953072196

http://orcid.org/0000-0002-6968-7969

okonrad@univates.br

\section{Referencing this:}

FEITOSA, A. K.; BARDEN, J. E.; KONRAD, O.. A relação entre a geração de resíduos sólidos domiciliares e os estratos socioeconômicos. Revista Ibero Americana de Ciências Ambientais, v.9, n.4, p.379-392, 2018. DOI: http://doi.org/10.6008/CBPC2179-6858.2018.004.0030 


\section{INTRODUÇÃO}

O crescimento na quantidade e complexidade dos Resíduos Sólidos Urbanos (RSU) tem decorrido do desenvolvimento econômico, da urbanização e aumento dos padrões de consumo da população (CHEN et al., 2016), culminando em graves problemas sanitários, especialmente registrados em países em desenvolvimento (DIAS et al., 2012).

Neste contexto, constata-se que conhecer a quantificação e caracterização dos RSU é primordial para as estratégias de gestão e manejo (KARAK et al., 2012; MELO et al., 2009), além de subsidiar o planejamento de programas de educação ambiental, que podem contribuir para a redução na geração de resíduos, organização da coleta, bem como melhores alternativas de destinação final (KONRAD et al., 2015).

Os "estudos sobre geração per capita de resíduos sólidos possibilitam gerar informações que possam servir como comparativo de valores em outros locais, ou auxiliar na gestão dos resíduos sólidos domésticos em municípios que ainda não tenham realizado estudos desse gênero" (KONRAD et al., 2015). Considerando que as questões socioeconômicas também têm relevância na geração de Resíduos Sólidos Urbanos, Habitzreuter (2008), em seu estudo na região de Santa Maria/RS, percebeu heterogeneidade na massa dos resíduos sólidos coletados, em função das diferenças socioeconômicas da região.

Costa et al. (2012), com o objetivo de identificar a composição gravimétrica dos resíduos sólidos domiciliares no município de Salinas, em Minas Gerais, apresentaram o perfil socioeconômico dos moradores e a correlação das características socioeconômicas com a composição gravimétrica dos resíduos. Dentre os resultados encontrados, destaca-se a maior ocorrência de material com potencial reciclável nos setores com famílias de maior poder aquisitivo e grau instrucional mais elevado. Já nos setores periféricos, com a predominância de famílias de baixa renda familiar e menor nível instrucional, há maiores percentuais de matéria orgânica.

Ribeiro et al. (2014) afirmaram que as características gravimétricas e a quantidade de resíduos gerados em uma comunidade servem de parâmetro de comparação entre distintas regiões. Para os autores, "em geral, economias mais avançadas, em termos da industrialização e produção, geram maior quantidade de resíduos por habitante". Validando este pensamento, conforme Martínez (2015), há uma relação existente entre as grandes cidades e uma maior produção de resíduos sólidos, em comparação com cidades pequenas.

Constata-se assim, que as diferenças socioeconômicas, bem como as questões culturais da população, exercem influência na composição gravimétrica e quantidade de resíduos gerados. Diante do exposto, o artigo tem por objetivo analisar a geração de resíduos sólidos domiciliares e a relação com os estratos socioeconômicos, na cidade de Juazeiro do Norte, Ceará.

\section{METODOLOGIA}

Para a realização deste estudo, inicialmente, foi identificada a renda domiciliar mensal e o número de domicílios de cada bairro da cidade de Juazeiro do Norte. Posteriormente, os bairros foram agrupados em 
estratos socioeconômicos, considerando a classificação da ABEP - Associação Brasileira de Empresas de Pesquisa (Tabela 1).

Tabela 1: Critério de Classificação de Estrato Socioeconômico.

\begin{tabular}{|l|l|}
\hline Estrato Socioeconômico & Renda Médica Domiciliar Mensal R\$ (2010) \\
\hline A1 & 12.926 \\
\hline A2 & 8.418 \\
\hline B1 & 4.418 \\
\hline B2 & 2.565 \\
\hline C1 & 1.541 \\
\hline C2 & 1.024 \\
\hline D & 714 \\
\hline E & 477 \\
\hline
\end{tabular}

Fonte: Adaptado de ABEP (2012).

Em 2014, foram divulgadas alterações nos estratos socioeconômicos, que reduziram-se a 6 (seis) níveis ( $A, B 1, B 2, C 1, C 2, D E)$, contudo, salienta-se que este estudo considera os critérios de classificação da ABEP alusivos ao ano 2010, disponibilizados na publicação Critério Brasil do ano 2012 da mesma instituição, pois não foi possível identificar dados recentes de renda domiciliar mensal para os bairros da cidade de Juazeiro do Norte. As informações disponíveis são oriundas do Censo 2010 do IBGE. Com relação ao número de domicílios, a Secretaria de Gestão do município apresentou dados do cadastro imobiliário, atualizado em janeiro de 2016, porém para alinhamento das informações, optou-se por utilizar os dados de 2010, que têm como fonte o último Censo realizado pelo IBGE. A metodologia de desenvolvimento do Critério Brasil da ABEP está baseada na POF - Pesquisa de Orçamento Familiar do IBGE (ABEP, 2015). Na Tabela 2, estão relacionados os bairros da cidade de Juazeiro do Norte, o número de domicílios, a renda domiciliar mensal e o estrato socioeconômico no qual o bairro está compreendido.

Dentre os bairros existentes, foram selecionados, de cada estrato socioeconômico, aqueles com maior número de domicílios para identificação da amostra de participação na análise gravimétrica dos resíduos domiciliares, quais sejam: Bairro Lagoa Seca, da classe B1; Bairro Salesianos, da classe B2; Bairro Pirajá, da classe C1; e, Bairro João Cabral, da classe C2. Os bairros das classes A2 e D, Planalto e Horto respectivamente, foram diretamente selecionados, tendo em vista serem os únicos representantes destes estratos no universo da amostra. Não houve bairro classificado no estrato socioeconômico A1.

Por questões operacionais de realização da pesquisa, foi, inicialmente, determinado o número de 50 domicílios para coleta dos resíduos. A partir deste dado, calculou-se o percentual proporcional referente ao número de domicílios de cada bairro selecionado do estrato socioeconômico que representa, por amostragem estratificada. Contudo, devido ao resultado para o bairro Planalto, único bairro enquadrado no estrato socioeconômico A2, com apenas 1 domicílio para amostra, optou-se por aumentar para 3 o número de domicílios participantes deste bairro, com a finalidade de evitar enviesamento na pesquisa.

Para os bairros Lagoa Seca e Salesianos, foi adotado o critério de arredondamento, visando estabelecer o número exato de domicílios e garantir melhor representatividade da amostra. Deste modo, o número total de domicílios para coleta e análise dos resíduos resultou em 53. A seleção dos domicílios a 
serem pesquisados ocorreu por Amostragem Aleatória Simples (GIL, 2008). De cada bairro, foi identificada a rua principal e os domicílios foram, então, sorteados para amostra e posterior cadastrado para a pesquisa.

Tabela 2: Bairros de Juazeiro do Norte por Estrato Socioeconômico.

\begin{tabular}{|c|c|c|c|}
\hline Bairro & $\begin{array}{l}\text { № Domicílios } \\
\text { (2010) }\end{array}$ & Renda Média Domiciliar Mensal, R\$ (2010) & Estrato Socioeconômico \\
\hline Planalto & 109 & 8745,44 & $\mathrm{~A} 2$ \\
\hline Lagoa Seca & 1433 & 4365,37 & B1 \\
\hline Centro & 1763 & 2907,71 & $\mathrm{~B} 1$ \\
\hline Aeroporto & 258 & 2851,64 & B1 \\
\hline Cidade Universitária & 85 & 2811,19 & B1 \\
\hline Jardim Gonzaga & 1638 & 2579,73 & B1 \\
\hline Novo Juazeiro & 988 & 2055,49 & B2 \\
\hline São Miguel & 2424 & 1888,36 & $\mathrm{~B} 2$ \\
\hline Salgadinho & 349 & 1874,18 & $\mathrm{~B} 2$ \\
\hline Betolândia & 538 & 1714,51 & B2 \\
\hline Socorro & 708 & 1651,04 & B2 \\
\hline Tiradentes & 2720 & 1606,22 & $\mathrm{~B} 2$ \\
\hline São José & 2759 & 1605,46 & B2 \\
\hline Salesianos & 4017 & 1582,03 & B2 \\
\hline Santo Antônio & 1680 & 1539,33 & $\mathrm{C} 1$ \\
\hline Limoeiro & 3430 & 1514,8 & $\mathrm{C} 1$ \\
\hline Fátima & 1067 & 1457,57 & $\mathrm{C} 1$ \\
\hline José Geraldo da Cruz & 1239 & 1338,51 & $\mathrm{C} 1$ \\
\hline Leandro Bezerra de Menezes & 860 & 1328,33 & $\mathrm{C} 1$ \\
\hline Antônio Vieira & 1902 & 1251,41 & $\mathrm{C} 1$ \\
\hline Franciscanos & 3615 & 1237,42 & $\mathrm{C} 1$ \\
\hline Juvêncio Santana & 1160 & 1205,12 & $\mathrm{C} 1$ \\
\hline Brejo Seco & 219 & 1191,05 & $\mathrm{C} 1$ \\
\hline Triângulo & 2668 & 1185,75 & $\mathrm{C} 1$ \\
\hline Santa Teresa & 1962 & 1151,39 & $\mathrm{C} 1$ \\
\hline Pirajá & 4204 & 1122,25 & $\mathrm{C} 1$ \\
\hline Romeirão & 1977 & 1013,92 & $\mathrm{C} 2$ \\
\hline Timbaúba & 3455 & 938,96 & $\mathrm{C} 2$ \\
\hline Pio XII & 3149 & 920,37 & $\mathrm{C} 2$ \\
\hline Carité & 263 & 784,47 & $\mathrm{C} 2$ \\
\hline João Cabral & 4809 & 775,22 & $\mathrm{C} 2$ \\
\hline Pedrinhas & 2552 & 772,3 & $\mathrm{C} 2$ \\
\hline Campo Alegre & 526 & 771,76 & $\mathrm{C} 2$ \\
\hline Frei Damião & 3864 & 763,22 & $\mathrm{C} 2$ \\
\hline Três Marias & 570 & 745,5 & $\mathrm{C} 2$ \\
\hline Horto & 1325 & 671,26 & $\mathrm{D}$ \\
\hline
\end{tabular}

Fonte: ABEP (2012); IBGE (2010).

Durante os cadastros dos domicílios, houve a substituição de dois bairros: o bairro Pirajá foi substituído pelo Limoeiro, no estrato socioeconômico $\mathrm{C} 1$, pois o barro Pirajá tem forte característica comercial e o foco da análise deste estudo eram resíduos domiciliares; e o bairro João Cabral foi substituído pelo Pio XII, pertencentes ao estrato C2, tendo o bairro Pio XII melhor facilidade de acesso. As quantidades de domicílios permaneceram inalteradas para garantir a representatividade do estrato socioeconômico na amostra definida (Tabela 3). A Figura 1 apresenta a localização geográfica dos bairros definidos na amostra e seus respectivos estratos socioeconômicos.

A coleta dos resíduos ocorreu, de porta em porta, de segunda a sexta-feira, durante o ano de 2016, nos períodos: 04 a 08 de abril; 20 a 24 de junho; e, 15 a 19 de agosto. Em cada dia da semana, os 53 domicílios tiveram seus resíduos coletados, resultando em 15 coletas por domicílio participante. Salienta-se que as 
datas foram selecionadas considerando-se a ausência de épocas festividades e férias escolares para evitar distorções de sazonalidade.

Tabela 3: Número de residências e habitantes cadastrados por Bairro.

\begin{tabular}{|l|l|l|l|}
\hline Bairro & Estrato Socioeconômico & Domicílios & Habitantes \\
\hline Planalto & A2 & 3 & 09 \\
\hline Lagoa Seca & B1 & 5 & 17 \\
\hline Salesianos & B2 & 13 & 53 \\
\hline Limoeiro & C1 & 13 & 38 \\
\hline Pio XII & C2 & 15 & 64 \\
\hline Horto & D & 4 & 12 \\
\hline TOTAL & - & 53 & 193 \\
\hline
\end{tabular}

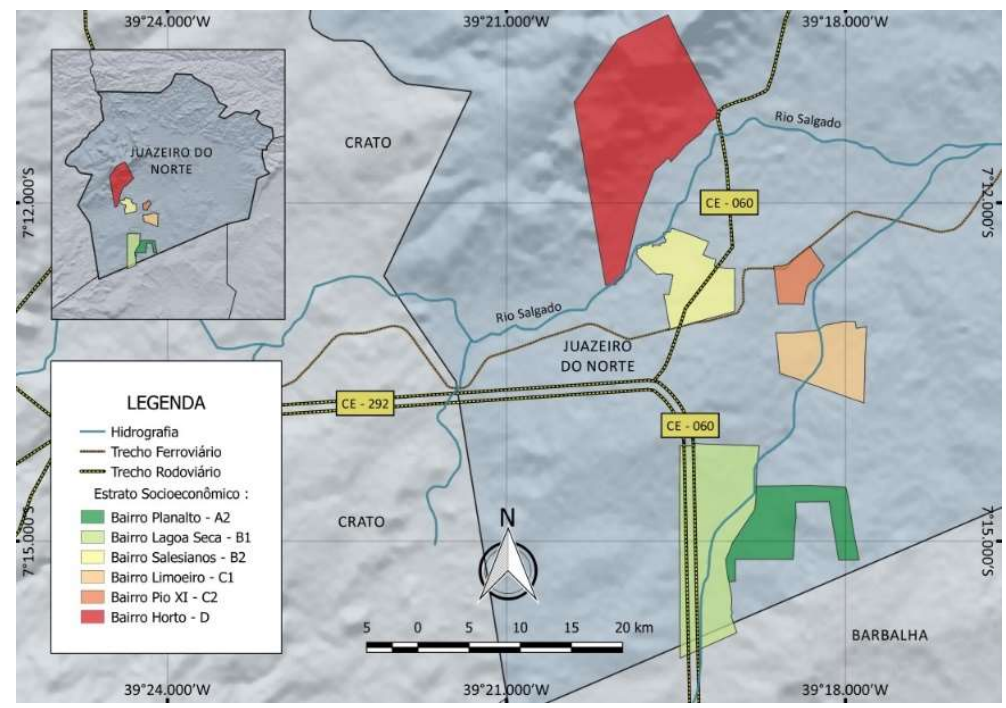

Figura 1: Localização dos bairros pesquisados.

Os meses de realização do estudo respeitam também as diferentes condições climáticas da cidade. Março e abril são meses chuvosos, com precipitação média de 243 mm em 2015, de acordo com o portal da FUNCEME - Fundação Cearense de Meteorologia e Recursos Hídricos (2015). O mês de Junho apresenta temperaturas brandas, entre $25{ }^{\circ} \mathrm{C}$ e 26 으, observadas no período de 2003 a 2012. De agosto a dezembro, o município possui temperaturas mais elevadas, apresentando a maior média mensal de temperatura, 35ㄷ, para o mês de novembro, segundo dados do ICEA - Instituto de Controle do Espaço Aéreo (ICEA, 2016). Em virtude das festividades de romarias locais ocorridas nos meses de setembro e novembro, e por ocasião do período eleitoral no mês de outubro de 2016, optou-se por realizar a terceira fase da análise gravimétrica no mês de agosto.

Após as coletas, foram analisadas as seguintes propriedades dos resíduos coletados: O cálculo da Geração per capita diária, dividindo-se a massa do resíduo coletado pela população atendida (kg/hab/dia); A massa específica, através do valor da soma das amostras obtidas em quilogramas dividido pelo volume $\left(\mathrm{kg} / \mathrm{m}^{3}\right)$; $\mathrm{E}$, a composição gravimétrica dos resíduos, que consiste em relacionar a massa de cada componente presente na amostra e a massa total da amostra de resíduos analisados.

Para análise dos resíduos, os seguintes elementos foram utilizados: seis tambores plásticos com capacidade de 120 litros para a coleta porta-a-porta, sacos plásticos de 100 litros, lona plástica, balanças 
digitais (de precisão, com capacidade de até $5 \mathrm{~kg}$, e de chão, com capacidade para até $180 \mathrm{~kg}$ ), uma mesa de separação, veículo para transportar os resíduos de cada bairro e equipamento de proteção individual.

\section{RESULTADOS E DISCUSSÃO}

No mês de abril, foram coletados $473,81 \mathrm{~kg}$ de resíduos nos bairros participantes, considerando 254 amostras de domicílios. No período de 20 a 24 de junho de 2016, foram coletados 396,13 kg, provenientes de 236 amostras de domicílios. No terceiro, e último, período, foram coletados $401,14 \mathrm{~kg}$, em 237 coletas realizadas (Tabela 4).

Tabela 4: Massa coletada de Resíduos Domiciliares em Juazeiro do Norte.

\begin{tabular}{|l|l|l|l|l|l|}
\hline Período & Domicílios & Coletas & Habitantes & Média de Habitante por domicílio & Massa RSD coletada (kg) \\
\hline Abril & 53 & 254 & 928 & 3,65 & 473,81 \\
\hline Junho & 53 & 236 & 887 & 3,76 & 396,13 \\
\hline Agosto & 53 & 237 & 875 & 3,69 & 401,14 \\
\hline Total & 53 & 727 & 2690 & 3,70 & $1.271,08$ \\
\hline
\end{tabular}

A respeito do número de habitantes e de coletas realizadas, houve variações devido a eventualidades de não participação de alguns domicílios, que se encontravam fechados no momento da coleta. Observandose a média de habitante por domicílio encontrada nas três campanhas, constata-se que os resultados são superiores à média de moradores dos lares brasileiros, que era de 3,3, segundo o Censo Demográfico de 2010 (IBGE, 2010).

\section{Análise da Variabilidade dos Resíduos Sólidos Domiciliares (RSD)}

No tocante à análise da variabilidade da composição gravimétrica, os resíduos coletados foram segregados, de acordo com sua tipologia, com a finalidade de estabelecer a caracterização dos mesmos, tendo como base a massa dos resíduos. Os resultados estão dispostos na Tabela 5. Observa-se que houve uma predominância de resíduos orgânicos nas amostras coletadas durante as três campanhas para o município de Juazeiro do Norte, atingindo os percentuais de 58,57\%, 58,29\% e 58,68\%, nos meses de abril, junho e agosto, respectivamente. Em estudos similares, Albertin et al. (2011) encontraram 63\% de resíduos orgânicos no município de São Jorge do Ivaí, Paraná. Outros estudos apresentam percentuais de materiais orgânicos próximos aos 50\% do total de resíduos sólidos coletados (DURÁN MORENO et al., 2013; REZENDE et al., 2013).

Em função da elevada quantidade de resíduos orgânicos, no presente estudo, destaca-se que seria apropriado o investimento em campanhas de orientação para a população relativamente ao desperdício de alimentos, diminuindo, deste modo, a quantidade de resíduos gerados em âmbito domiciliar (SALEMDEEB et al., 2017; WILLERSINN et al., 2017). Além disso, no que se refere ao tratamento dos resíduos orgânicos, é conveniente implementar uma infraestrutura de compostagem para a valorização destes resíduos, culminando em benefícios ambientais e econômicos (HÉNAULT-ETHIER et al., 2017).

Zorpas et al. (2017) afirmam que, a partir dos resíduos orgânicos, é possível produzir um fertilizante natural de alta qualidade. Adicionalmente, Walker et al. (2017) apontam a produção de biogás a partir dos 
resíduos orgânicos como uma alternativa para o enfrentamento do problema do gerenciamento da maior parcela dos resíduos sólidos domiciliares.

Tabela 5: Análise da Variabilidade dos Resíduos Domiciliares em Juazeiro do Norte

\begin{tabular}{|l|l|l|l|l|}
\hline Tipo de resíduo & Abril (\%) & Junho (\%) & Agosto (\%) & Média (\%) \\
\hline Materiais orgânicos & 58,57 & 58,29 & 58,68 & 58,51 \\
\hline Plástico filme & 3,57 & 5,53 & 5,08 & 4,73 \\
\hline Plástico rígido & 2,27 & 3,44 & 2,40 & 2,70 \\
\hline PET & 0,99 & 1,30 & 1,20 & 1,16 \\
\hline Papel/jornal & 1,61 & 2,02 & 2,74 & 2,12 \\
\hline Papelão & 2,79 & 2,85 & 2,76 & 2,80 \\
\hline Tetra pak & 0,74 & 0,64 & 0,47 & 0,62 \\
\hline Vidro & 1,37 & 1,02 & 2,57 & 1,65 \\
\hline Metal/alumínio & 1,02 & 1,25 & 0,82 & 1,03 \\
\hline Isopor & 0,16 & 0,15 & 0,26 & 0,19 \\
\hline $\begin{array}{l}\text { Embalagens } \\
\text { metalizadas }\end{array}$ & $*$ & 0,57 & 0,56 & 0,38 \\
\hline Fraldas & 8,44 & 10,39 & 10,85 & 9,89 \\
\hline Papel sanitário & 3,62 & 3,57 & 3,27 & 3,49 \\
\hline Trapos & 2,05 & 2,88 & 2,52 & 2,48 \\
\hline Madeira & 0,06 & 0,23 & 0,21 & 0,17 \\
\hline Rejeito & 9,77 & 4,95 & 3,55 & 6,09 \\
\hline Borracha & 0,15 & 0,49 & 0,22 \\
\hline Resíduos Especiais & 0,33 & 0,35 & 0,22 & 0,30 \\
\hline $\begin{array}{l}\text { Resíduos } \\
\text { Construção Civil }\end{array}$ & 2,28 & 0,16 & 1,34 & 1,26 \\
\hline Mela & 0,35 & 0,26 & 0,01 & 0,21 \\
\hline Total & 100 & 100 & 100 & 100 \\
\hline
\end{tabular}

*Na primeira campanha, as embalagens metalizadas foram incorporadas na categoria rejeito.

Com relação aos materiais com potencial reciclável, foi possível identificar maior geração de plásticos e papelão, dentre os materiais coletados. 0 total de materiais recicláveis oscilou entre $14,71 \%$ do total de resíduos gerados na campanha de abril, 18,31\% na campanha de junho e 18,05\% na campanha de agosto, com média de 17,02\%. A este respeito, a literatura apresenta uma variabilidade de potencial de reaproveitamento de materiais recicláveis, variando entre $8,87 \%$ e $76 \%$ dos resíduos coletados em diversos estudos (ALBERTIN et al., 2011; COSTA et al., 2012; CUSSIOL et al., 2006; KONRAD et al., 2010; REZENDE et al., 2013; ROSALES-FLORES et al., 2013; SOUTO et al., 2013; TEIXEIRA JÚNIOR et al., 2012).

Dentre os tipos de resíduos, foram adicionados: o 'Mela' (que corresponde a um tipo de borracha PVC, utilizada na confecção de chinelos e que possui uma aceitação na reciclagem local, por isso, optou-se por manter esta categoria separada. Sobre os resíduos rejeitos gerados ao longo das três campanhas de análises, foi encontrada uma média de $24,48 \%$, sendo $26,76 \%$ em abril, $23,40 \%$ em junho e $23,37 \%$ em agosto. Foram considerados: esponja, palha de aço de cozinha, algodão, vela, cabelo, pelo de cachorro, caixa de fósforo, papel ou plástico muito sujos em função do descarte misturado com resíduos orgânicos e palito de fósforo/pirulito.

Alguns resíduos foram segregados em uma categoria própria, a fim de identificar os percentuais gerados, porém, ao final da análise foram classificados como rejeitos: resíduos de construção civil; madeira; borracha; fraldas; papel sanitário; trapos; resíduos especiais, que exigem tratamentos e destinações específicas como medicamentos, pilhas, fios, equipamentos eletrônicos e perfurocortantes; os resíduos isopor e embalagens metalizadas de alimentos, conhecidas como BOPP (Bi-axially Oriented Polypropylene), 
porque possuem pouca inserção no mercado de reciclagem em função do seu baixo valor comercial e da falta de interesse na reciclagem destes materiais.

\section{Caracterização dos Resíduos Sólidos Domiciliares por Estrato Socioeconômico}

De acordo com a amostra de resíduos coletada e analisada, durante as três campanhas de análise gravimétrica, foi possível realizar a caracterização em cada bairro estudado (Tabela 6).

Tabela 6: Caracterização dos Resíduos Sólidos Domiciliares.

\begin{tabular}{|c|c|c|c|c|c|c|}
\hline Bairro & Estrato & Período & Reciclável \% & Orgânico \% & Rejeito \% & Total \% \\
\hline \multirow[t]{3}{*}{ Planalto } & \multirow[t]{3}{*}{$\mathrm{A} 2$} & Abril & 23,23 & 61,33 & 15,44 & 100 \\
\hline & & Junho & 41,32 & 40,78 & 17,90 & 100 \\
\hline & & Agosto & 30,38 & 50,09 & 19,53 & 100 \\
\hline \multirow[t]{3}{*}{ Lagoa Seca } & \multirow[t]{3}{*}{ B1 } & Abril & 15,65 & 67,80 & 16,55 & 100 \\
\hline & & Junho & 21,17 & 64,13 & 14,70 & 100 \\
\hline & & Agosto & 16,97 & 75,02 & 8,01 & 100 \\
\hline \multirow[t]{3}{*}{ Salesianos } & \multirow[t]{3}{*}{ B2 } & Abril & 17,81 & 59,62 & 22,57 & 100 \\
\hline & & Junho & 17,16 & 59,96 & 22,88 & 100 \\
\hline & & Agosto & 17,28 & 63,36 & 19,36 & 100 \\
\hline \multirow[t]{3}{*}{ Limoeiro } & \multirow[t]{3}{*}{$\mathrm{C} 1$} & Abril & 11,61 & 48,20 & 40,19 & 100 \\
\hline & & Junho & 17,02 & 51,92 & 31,06 & 100 \\
\hline & & Agosto & 14,95 & 52,45 & 32,60 & 100 \\
\hline \multirow[t]{3}{*}{ Pio XII } & \multirow[t]{3}{*}{$\mathrm{C} 2$} & Abril & 11,15 & 62,40 & 26,45 & 100 \\
\hline & & Junho & 15,85 & 60,86 & 23,29 & 100 \\
\hline & & Agosto & 14,22 & 59,83 & 25,95 & 100 \\
\hline \multirow[t]{3}{*}{ Horto } & \multirow[t]{3}{*}{$\mathrm{D}$} & Abril & 19,56 & 53,34 & 27,10 & 100 \\
\hline & & Junho & 30,26 & 56,65 & 13,09 & 100 \\
\hline & & Agosto & 23,39 & 60,02 & 16,59 & 100 \\
\hline
\end{tabular}

Sobre a geração de Resíduos Orgânicos, tem-se o maior registro de geração no bairro Lagoa Seca, bairro pertencente ao estrato socioeconômico B1, durante o mês de agosto, equivalendo a 75,02\% dos resíduos gerados. O bairro que apresentou a menor geração de resíduos orgânicos foi o bairro Planalto, do estrato $A 2$, com 40,78\% do total de resíduos, no mês de junho.

De acordo com os valores médios de geração dos resíduos orgânicos, destacaram-se os resultados dos bairros Lagoa Seca, com média de 68,98\%, Pio XII, com 61,02\% e Salesianos, com 60,98\%, pertencentes aos estratos B1, C2 e B2, respectivamente. O bairro Horto, pertencente ao estrato $D$, obteve uma média de 56,67\% de resíduos orgânicos. Observa-se que, para a amostra analisada, não é possível estabelecer uma relação linear entre os estratos socioeconômicos e a geração de resíduos orgânicos, contrariando os resultados de estudos similares, que evidenciaram maior quantidade de matéria orgânica dentre os bairros de menor poder aquisitivo (COSTA et al., 2012; FRANCO, 2012) ou indicaram uma relação linear positiva entre renda e taxa de geração de resíduos alimentares (THANH et al., 2010).

Analisando a composição dos resíduos com potencial reciclável por bairro gerador, pode-se afirmar que a geração de materiais com potencial reciclável foi maior no bairro Planalto, do estrato socioeconômico A2, com geração média de $31,64 \%$ dentre os resíduos coletados. A este respeito, corrobora o estudo de Franco (2012), que afirma ocorrer maior quantidade de descarte de recicláveis em domicílios de estrato socioeconômico A e B, em comparação com domicílios do estrato C. Estes resultados estão em consonância com a ideia de que o descarte de resíduos recicláveis, especialmente de produtos descartáveis e embalagens, 
ocorre em maior quantidade em domicílios de estratos com maior poder aquisitivo (ARBULÚ et al., 2015; COSTA et al., 2012).

Não obstante, curiosamente, destacou-se nos resultados o desempenho do bairro Horto na geração de materiais recicláveis. $O$ referido bairro, pertencente ao estrato $D$, obteve uma geração superior aos bairros pertencentes aos estratos B e C, tendo registrado uma geração média de $24,40 \%$ de resíduos com potencial reciclável. Esta realidade pode ser justificada pela afirmação de Campos (2012), em que "o aumento do número de postos de trabalho e da renda das famílias das classes D e E [...] tem favorecido a mobilidade social e o aumento do consumo". A isto somam-se as hipóteses de aumento da geração de resíduos apresentadas pela autora: facilidade na obtenção de crédito para o consumo; estímulo frenético ao consumo pelos veículos de comunicação; e uso indiscriminado de produtos descartáveis (CAMPOS, 2012). Medeiros, et al. (2015), salientam que:

Nas áreas onde vive a população de renda mais baixa a coleta de RSD pode não ser tão eficiente quanto a que ocorre nos bairros de maior renda, devido à menor frequência da coleta, o que pode induzir ao descarte de parcela dos resíduos em terrenos baldios, reduzindo a massa coletada de RSD.

Esta afirmativa pode justificar os baixos níveis de resíduos registrados na literatura para os bairros de estratos socioeconômicos D e E. Ressalta-se que, no presente estudo, a coleta foi realizada porta-a-porta, em domicílios previamente cadastrados, antes do descarte para a coleta convencional. Assim, os percentuais encontrados para o bairro Horto representam a realidade da amostra analisada.

Quanto à geração de rejeitos, foram encontrados menores índices percentuais nos bairros Lagoa Seca, com média de 13,09\%, e Planalto, com 17,62\%, bairros que possuem maiores estratos socioeconômicos. A este respeito, salienta-se a forma de separação dos resíduos adotada pelos habitantes de cada bairro. Percebeu-se que os domicílios de bairros correspondentes aos estratos socioeconômicos mais elevados possuíam a preocupação de segregar seus resíduos entre secos e úmidos, o que contribuiu para uma maior qualidade dos recicláveis e, consequentemente, menor percentual de rejeitos.

Neste sentido, observa-se a necessidade de investimento em educação ambiental para a população, que deveria ser orientada sobre a separação adequada dos resíduos no momento do descarte, observandose os problemas ambientais decorrentes que afetam o meio ambiente e a saúde humana (MORAR; BUCUR, 2017). Adicionalmente, registra-se a falta de um apropriado sistema de coleta, transporte e destinação de resíduos, visando a recuperação dos materiais (LIU et al., 2017; ZENG et al., 2016), além da carência de empreendimentos especializados na indústria da reciclagem atuantes na região.

Deste modo, para que exista um melhor aproveitamento dos resíduos com potencial reciclável, e consequentemente uma menor geração de rejeitos, é necessário que todos os sujeitos envolvidos (setor público, empresarial e comunidade) compartilhem responsabilidades e consciência coletiva para o fortalecimento de uma eficiente gestão de resíduos (INOUE et al., 2016). 
Volume e Massa Específica dos Resíduos Sólidos Domiciliares

Nesta seção dos resultados, estão apresentados o volume e a massa específica dos resíduos coletados e analisados. Na Tabela 7, contam os volumes agrupados nas classes de resíduos recicláveis, orgânicos e rejeitos.

Tabela 7: Volume dos Resíduos Gerados.

\begin{tabular}{|l|l|l|l|l|}
\hline Período & Classe & Volume (I) & Volume $\left.\mathbf{( m}^{\mathbf{3}}\right)$ & \% Volume \\
\hline \multirow{5}{*}{ Abril } & Reciclável & 878,59 & 0,879 & 31,88 \\
\cline { 2 - 5 } & Orgânico & 450,00 & 0,450 & 16,29 \\
\cline { 2 - 5 } & Rejeito & 414,41 & 0,414 & 14,98 \\
\cline { 2 - 5 } & Indiferenciado & 1020,00 & 0,102 & 36,92 \\
\hline \multirow{4}{*}{ Jutal } & - & $2763,00^{*}$ & 2,763 & 100 \\
& Reciclável & 1901,00 & 1,901 & 56,76 \\
\cline { 2 - 5 } & Orgânico & 635,50 & 0,636 & 18,99 \\
\cline { 2 - 5 } & Rejeito & 812,10 & 0,812 & 24,25 \\
\hline Total & - & 3348,60 & 3,349 & 100 \\
\hline \multirow{5}{*}{ Agosto } & Reciclável & 1876,00 & 1,876 & 57,70 \\
\cline { 2 - 5 } & Orgânico & 617,00 & 0,617 & 18,98 \\
\cline { 2 - 5 } & Rejeito & 757,50 & 0,758 & 23,32 \\
\hline Total & - & 3250,50 & 3,251 & 100 \\
\hline
\end{tabular}

*Durante a campanha de abril, o volume diferenciado por classe de resíduos foi calculado somente a partir das amostras coletadas de 6 a 8 de abril. 0 volume total compreende o período de 4 a 8 do mês.

Com base na Tabela 7, observa-se que o volume de materiais com potencial reciclável foi o maior, tendo registrado os percentuais de $56,76 \%$ e $57,70 \%$, em junho e agosto, respectivamente. Os resíduos rejeitos apresentaram a segunda maior média de volume, com 20,85\% do total de resíduos analisados. Por fim, os materiais orgânicos apresentaram uma média de volume de $18,06 \%$ do total de resíduos.

Com relação à massa específica dos resíduos (Tabela 8), os resultados para as três campanhas de análises foram: $171,48 \mathrm{~kg} / \mathrm{m}^{3}$, considerando a massa de $473,81 \mathrm{~kg}$ e o volume de $2,763 \mathrm{~m}^{3}$, no mês de abril; $118,28 \mathrm{~kg} / \mathrm{m}^{3}$, considerando $396,13 \mathrm{~kg}$ de massa e 3,349 $\mathrm{m}^{3}$ de volume, no mês de junho; e, $123,39 \mathrm{~kg} / \mathrm{m}^{3}$, considerando $401,14 \mathrm{~kg}$ de massa e $3,251 \mathrm{~m}^{3}$ de volume, no mês de agosto.

Tabela 8: Massa Específica dos Resíduos Sólidos Domiciliares.

\begin{tabular}{|c|c|c|c|c|c|c|c|c|}
\hline & Bairro & Planalto & Lagoa Seca & Salesianos & Limoeiro & Pio XII & Horto & Total \\
\hline & Estrato & $\mathrm{A} 2$ & B1 & B2 & $\mathrm{C} 1$ & $\mathrm{C} 2$ & $\mathrm{D}$ & - \\
\hline \multirow[t]{3}{*}{ Abril } & Massa $(\mathrm{kg})$ & 22,94 & 64,58 & 135,91 & 89,58 & 139,36 & 21,44 & 473,81 \\
\hline & Volume $\left(\mathrm{m}^{3}\right)$ & 0,175 & 0,405 & 0,800 & 0,520 & 0,655 & 0,208 & 2.763 \\
\hline & $\begin{array}{l}\text { Massa Específica } \\
\left(\mathrm{kg} / \mathrm{m}^{3}\right)\end{array}$ & 131,09 & 159,46 & 169,89 & 172,27 & 212,76 & 103,08 & 171,48 \\
\hline \multirow[t]{3}{*}{ Junho } & Massa $(\mathrm{kg})$ & 16,32 & 50,95 & 90,18 & 91,94 & 128,81 & 17,93 & 396,13 \\
\hline & Volume $\left(\mathrm{m}^{3}\right)$ & 0,169 & 0,545 & 0,765 & 0,662 & 0,933 & 0,275 & 3,349 \\
\hline & $\begin{array}{l}\text { Massa Específica } \\
\left(\mathrm{kg} / \mathrm{m}^{3}\right)\end{array}$ & 96,85 & 93,49 & 117,88 & 138,88 & 138,06 & 65,19 & 118,28 \\
\hline \multirow[t]{3}{*}{ Agosto } & Massa (kg) & 29,90 & 54,33 & 87,61 & 93,70 & 120,28 & 15,32 & 401,14 \\
\hline & Volume $\left(\mathrm{m}^{3}\right)$ & 0,369 & 0,514 & 0,728 & 0,590 & 0,785 & 0,265 & 3,251 \\
\hline & $\begin{array}{l}\text { Massa Específica } \\
\left(\mathrm{kg} / \mathrm{m}^{3}\right)\end{array}$ & 81,13 & 105,71 & 120,34 & 158,82 & 153,22 & 57,80 & 123,39 \\
\hline
\end{tabular}

Observa-se que, durante a campanha de abril, a massa específica variou entre $103,08 \mathrm{~kg} / \mathrm{m}^{3}$ no bairro Horto, estrato $D$, e $212,76 \mathrm{~kg} / \mathrm{m}^{3}$ no bairro Pio XII, estrato C2. Nas campanhas de junho e agosto, a maior massa específica registrada foi encontrada no bairro Limoeiro, no estrato $C 1$, com $138,88 \mathrm{~kg} / \mathrm{m}^{3}$ em junho e $158,82 \mathrm{~kg} / \mathrm{m}^{3}$ no mês de agosto. Ao longo das três campanhas, a massa específica dos bairros estudados 
oscilou entre $57,80 \mathrm{~kg} / \mathrm{m}^{3}$, no bairro Horto, no mês de agosto, e $212,76 \mathrm{~kg} / \mathrm{m}^{3}$, no bairro Pio XII, no mês de abril.

O bairro Horto, no estrato socioeconômico $D$, registrou a menor massa específica nas três fases do estudo. Baseando-se neste dado, pode-se estimar que há geração de resíduo sólido com menos massa específica no bairro Horto, representando uma aparente redução do consumo. No entanto, esta informação pode representar também hábitos de consumo de produtos industrializados ou semiprocessados, que supostamente podem reduzir o peso específico dos resíduos. Neste último caso, há geração de resíduos sólidos mais complexos em função do tipo de embalagem, por exemplo isopor e tetra pak (CAMPOS, 2012). Pode-se inferir que esta informação corrobora com a geração de resíduos com potencial reciclável evidenciada, neste estudo, para o bairro Horto (item 3.2).

O conhecimento da massa específica é uma fundamental informação para o correto dimensionamento de equipamentos e instalações (CAPAZ et al., 2014). Pode-se afirmar que são dados necessários para o gerenciamento da coleta, transporte e disposição final dos resíduos, em relação à capacidade de infraestrutura do município de Juazeiro do Norte.

\section{Geração per capita diária de resíduos}

Para finalizar a análise, estabeleceu-se a variável que apresenta a geração per capita diária de resíduos ( $\mathrm{kg} /$ habitante/dia) da amostra, que resultou em 0,51 na campanha de abril, 0,45 em junho e 0,46 em agosto. Os resultados para os três períodos apresentaram uma média de 0,47 kg/hab/dia, mantendo-se abaixo da média nacional no diagnóstico do manejo de resíduos sólidos urbanos de 2014, que aponta 1,05 $\mathrm{kg} / \mathrm{hab} / \mathrm{dia}$ (BRASIL, 2016).

Salienta-se que, considerando-se o porte do município, a geração de resíduos sólidos pode variar. Para a realidade de Juazeiro do Norte, que em 2015 possuía uma população estimada em 266.022 habitantes, segundo dados do IBGE, os valores de geração per capita enquadram-se na faixa populacional 4 (entre 250.001 a 1.000 .000 habitantes), que apresenta para geração de resíduos per capita diária os valores: mínimo, 0,49; máximo, 1,94; e, médio, 0,99 (BRASIL, 2016). Em estudos de semelhantes, em outras regiões do Brasil, identificou-se a geração per capita diária de resíduos sólidos variando entre 0,46 e 1,60 kg/hab/dia (ALBERTIN et al., 2011; KONRAD et al., 2010; KONRAD et al., 2015; REZENDE et al., 2013; TEIXEIRA JÚNIOR, 2012). Foi possível estabelecer, ainda, a geração per capita diária de cada bairro, bem como a média dos três períodos (Tabela 9).

Tabela 9: Geração per capita diária por bairro.

\begin{tabular}{|l|l|l|l|l|l|}
\hline \multirow{2}{*}{ Bairro } & Geração per capita diária & \multicolumn{2}{l|}{} \\
\cline { 2 - 6 } & Estrato & Abril & Junho & Agosto & Média \\
\hline Planalto & A2 & 0,56 & 0,37 & 0,77 & 0,57 \\
\hline Lagoa Seca & B1 & 0,76 & 0,66 & 0,67 & 0,70 \\
\hline Salesianos & B2 & 0,51 & 0,35 & 0,37 & 0,41 \\
\hline Limoeiro & C1 & 0,48 & 0,57 & 0,60 & 0,55 \\
\hline Pio XII & C2 & 0,45 & 0,42 & 0,39 & 0,42 \\
\hline Horto & D & 0,50 & 0,42 & 0,29 & 0,40 \\
\hline Juazeiro do Norte & - & 0,51 & 0,45 & 0,46 & 0,47 \\
\hline
\end{tabular}


A média de geração per capita diária nos bairros para as amostras de estudo, oscilou entre 0,40 $\mathrm{kg} / \mathrm{hab} / \mathrm{dia}$, no bairro Horto, e $0,70 \mathrm{~kg} / \mathrm{hab} / \mathrm{dia}$, no bairro Lagoa Seca. Considerando o estrato socioeconômico dos bairros da amostra, observa-se que há uma maior quantidade de resíduos gerados no bairro Lagoa Seca, no estrato B1, seguido do bairro Planalto, no estrato A2, e do bairro Limoeiro, no estrato C1, com médias de geração per capita diária de 0,70, 0,57 e 0,55, respectivamente.

Em consonância com estes resultados, Franco (2012), em estudo realizado no sul de Minas Gerais, no sudeste do Brasil, encontrou uma maior geração per capita diária de RSD atribuída a domicílios pertencentes ao estrato socioeconômico $B$, seguido do $\mathrm{A}$ e, por fim, do $\mathrm{C}$, resultando, este último, na menor geração. Xiao et al. (2015), encontraram resultados similares, relacionando a geração de resíduos sólidos urbanos com a renda de unidades familiares na China.

Enfatiza-se que estas informações são consistentes com a Teoria da Curva Ambiental de Kuznets, que apresenta uma hipótese de relação entre a degradação ambiental, neste caso representada pela geração de resíduos sólidos urbanos, e a renda, assumindo uma curva em forma de ' $U$ ' invertido. Tem-se que a geração de resíduos aumenta até determinado ponto (ou nível de renda), mas, finalmente, diminui com o nível de renda mais elevado (ARBULÚ et al., 2015; XIAO et al., 2015). A este respeito, Campos (2012) afirma que "as variações do nível de ocupação da população, da massa de rendimento e do rendimento médio real são fatores relevantes para a compreensão da evolução do consumo e da consequente geração de resíduos sólidos".

\section{CONCLUSÕES}

De acordo com os resultados deste estudo, a caracterização gravimétrica dos resíduos sólidos demonstrou que a fração quantitativa mais significativa dos resíduos gerados em Juazeiro do Norte era constituída de matéria orgânica (restos de alimentos, cascas de frutos, borra de café e folhas). Com relação aos materiais com potencial reciclável, foi possível identificar maior geração de plásticos e papelão. Considerando-se que, entre resíduos secos e orgânicos, apresentam-se cerca de 75,5\% dos resíduos coletados e analisados, pode-se inferir a respeito da viabilidade de implantação de um sistema de coleta diferenciada e posterior valorização de resíduos sólidos domiciliares.

Ressalta-se que a maior parte dos resíduos orgânicos foi encontrada no bairro Lagoa Seca, pertencente ao estrato socioeconômico B1, contrariando a literatura científica que apresenta congruência de maiores resultados de resíduos orgânicos em bairros de menor nível socioeconômico. Relativamente aos materiais com potencial reciclável, observou-se maior geração no bairro de estrato socioeconômico 'A2', em função do padrão de consumo de alimentos mais processados, indicando maior geração de resíduos de embalagens. Não obstante, o bairro de estrato ' $D$ ' obteve uma geração de materiais recicláveis superior aos bairros dos estratos ' $B$ ' e ' $C$ ', o que não era um resultado esperado, uma vez que se trata de um bairro com baixos rendimentos.

Em termos quantitativos, evidencia-se que a geração per capita de resíduos sólidos domiciliares apresenta uma relação positiva de acordo com o nível de renda do bairro gerador, porém diminui quando 
atinge o nível mais elevado de rendimentos, guardando semelhança empírica com a hipótese da Curva Ambiental de Kuznets. Salienta-se que a estratificação em perfis socioeconômicos é importante para investigar os padrões de geração de resíduos domésticos nos centros urbanos e contribuir com informações mais assertivas para o gerenciamento dos resíduos sólidos.

Em estudos futuros, poder-se-á analisar a hipótese da estrutura de uma curva de Kuznets entre a geração de resíduos e o nível de rendimentos dos estratos socioeconômicos. Adicionalmente, será possível identificar os fatores subjacentes à determinação da geração de resíduos domésticos para as áreas estudadas, tais como hábitos alimentares, estilo de vida e estrutura familiar.

\section{REFERÊNCIAS}

ABEP. Associação Brasileira de Empresas de Pesquisa. Critério de Classificação Econômica Brasil. 2012.

ABEP. Associação Brasileira de Empresas de Pesquisa. Critério de Classificação Econômica Brasil. 2015.

ALBERTIN, R. M.; MORAES, E.; SILVA, F. F. S.; CORVELONI, E. P. M.; ANGELIS NETO, G.; ANGELIS NETO, B.. Proposta de ampliação do programa de coleta seletiva para o município de São Jorge do Ivaí no Estado do Paraná. Revista Agro@mbiente On-line, Boa Vista, v.5, n.1, p.75-81, 2011.

ARBULÚ, I.; LOZANO, J.; REY-MAQUIEIRA, J.. Tourism and solid waste generation in Europe: A panel data assessment of the Environmental Kuznets Curve. Waste Management, v.46, p.628-636, 2015.

BRASIL. Ministério das Cidades. Sistema Nacional de Informações sobre Saneamento: diagnóstico do manejo de resíduos sólidos urbanos - 2014. Brasília: MCIDADES.SNSA, 2016.

CAMPOS, H. K. T.. Renda e evolução da geração per capita de resíduos sólidos no Brasil. Eng. Sanit. Ambient., Rio de Janeiro, v.17, n.2, p.171-180, 2012.

CAPAZ, R.; NOGUEIRA, L.. Ciências Ambientais para Engenharia. 1 ed. Elsevier: Rio de Janeiro, 2014.

CHEN, Y. C.; LO, S. L.. Evaluation of greenhouse gas emissions for several municipal solid waste management strategies. Journal of Cleaner Production, v.113, p.606-612, 2016.

COSTA, L. E. B.; COSTA, S. K.; REGO, N. A. C.; SILVA JUNIOR, M. F.. Gravimétrica dos Resíduos Sólidos Urbanos Domiciliares e Perfil Socioeconômico no Município de Salinas, Minas Gerais. Revista Ibero-Americana de Ciências Ambientais, v.3, n.1, 2012.

CUSSIOL, N. A. M.; ROCHA, G. H. T.; LANGE, L. C.. Quantificação dos resíduos potencialmente infectantes presentes nos resíduos sólidos urbanos da regional sul de Belo Horizonte, Minas Gerais, Brasil. Cad. Saúde Pública, Rio de Janeiro, v.22, n.6, p.1183-1191, 2006.

DIAS, D. M.; MARTINEZ, C. B.; BARROS, R. T. V.; MARCELO, L.. Modelo para estimativa da geração de resíduos sólidos domiciliares em centros urbanos a partir de variáveis socioeconômicas conjunturais. Eng. Sanit. Ambient., Rio de
Janeiro, v.17, n.3, p.325-332, 2012.

DURÁN MORENO, A.; GARCÉS RODRÍGUEZ, M.; VELASCO, A. R.; MARÍN ENRIQUEZ, J. C.; GUTIÉRREZ LARA, R.; MORENO GUTIÉRREZ, A.; DELGADILLO HERNÁNDEZ, N. A..

Características y análisis de composición de los residuos sólidos de la Ciudad de México. Rev. Int. Contam. Ambient, México, v.29, n.1, p.47-57, 2013.

FRANCO, C. S.. Caracterização Gravimétrica dos Resíduos Sólidos Domiciliares e Percepção dos Hábitos de Descarte no Sul de Minas Gerais. Dissertação (Mestrado em Engenharia Agrícola) - Universidade Federal de Lavras, Lavras, 2012.

FUNCEME. Chuva Mensal (mm). 2015.

GIL, A. C.. Métodos e técnicas de pesquisa social. 6 ed. Atlas Novo: São Paulo, 2008.

HABITZREUTER, M. T.. Análise da compactação gravimétrica dos resíduos sólidos urbanos (RSU) da Região de Santa Maria, pré e pós-triagem. Dissertação (Pós-graduação em Engenharia Civil) - Universidade Federal do Rio Grande do Sul, Porto Alegre, 2008.

HÉNAULT-ETHIER, L.; MARTIN, J. P.; HOUSSET, J.. A dynamic model for organic waste management in Quebec ( $D$ MOWIQ) as a tool to review environmental, societal and economic perspectives of a waste management policy. Waste Management, v.66, p.196-209, 2017.

IBGE. Instituto Brasileiro de Geografia e Estatística. Censo Demográfico 2010. 2010.

ICEA. Instituto de Controle do Espaço Aéreo. Temperatura de Referência do Juazeiro do Norte/CE. 2016

INOUE, C. Y. A.; RIBEIRO, T. M. M. L.. Padrões sustentáveis de produção e consumo: resíduos sólidos e os desafios de governança do global ao local. Journal of Global Studies, v.47, n.17, p.1-9, 2016.

KARAK, T.; BHAGAT, R. M.; BHATTACHARYYA, P.. Municipal solid waste generation, composition, and management: The World Scenario. Critical Reviews Environmental Science and Technology, v.42, n.15, p.1509-1630, 2012. 
KONRAD, O.; CASARIL, C. E.; SCHMITZ, M.. Estudo dos resíduos sólidos domésticos de Lajeado/RS pela caracterização gravimétrica. Destaques Acadêmicos, v.4, p.57-62, 2010.

KONRAD, O.; SILVA, G. R.; KONRAD, A. C.; HASAN, C.; MARDER, M.; SCHMEIER, N. P.. Geração per Capita de Resíduos Sólidos Domésticos na Zona Urbana de Municípios do Vale do Taquari/RS. Revista Educação Ambiental, v.53, Ano XIV, 2015.

LIU, G.; HAO, Y.; DONG, L.; ZHANG, Y.; ULGIATI, S.. An emergy-LCA analysis of municipal solid waste management. Resources, Conservation and Recycling, v.120, p.131-143, 2017.

MARTÍNEZ, N. M. J.. La gestión integral de residuos sólidos urbanos en México: entre la intención y la realidad. Letras Verdes. Revista Latinoamericana de Estudios Socioambientales, n.17, p.29-56, 2015.

MEDEIROS, J. E. S. F.; PAZ, A. R.; MORAIS JUNIOR, J. A. Análise da evolução e estimativa futura da massa coletada de resíduos sólidos domiciliares no município de João Pessoa e relação com outros indicadores de consumo. Eng. Sanit. Ambient., Rio de Janeiro, v.20, n.1, p.119-130, 2015.

MELO, L. A.; SAUTTER, K. D.; JANISSEK, P. R.. Estudo de cenários para o gerenciamento dos resíduos sólidos urbanos de Curitiba. Eng. Sanit. Ambient., Rio de Janeiro, v.14, n.4, p.551-558, 2009.

MORAR, F.; BUCUR, B.. Raising Awareness on Waste Generation and Collection. Procedia Engineering, v.181, p.452-458, 2017.

REZENDE, J. H.; CARBONI, M.; MURGEL, M. A. T.; CAPPS, A. L. A. P.; TEIXEIRA, H. L.. Composição gravimétrica e peso específico dos resíduos sólidos urbanos em Jaú (SP). Eng. Sanit. Ambient., Rio de Janeiro, v.18, n.1, p.1-8, 2013.

RIBEIRO, L. C. S.; FREITAS, L. F. S.; CARVALHO, J. T. A.; OLIVEIRA FILHO, J. D.. Aspectos econômicos e ambientais da reciclagem: um estudo exploratório nas cooperativas de catadores de material reciclável do Estado do Rio de Janeiro. Nova econ., Belo Horizonte, v.24, n.1, p.191-2014, 2014.

ROSALES-FLORES, M.; SALDAÑA-DURÁN, C.; TOLEDORAMÍREZ ,V.; MALDONADO, L.. Caracterización y potencial del reciclado de los residuos sólidos urbanos generados en el Instituto Tecnológico de Tepic, una institución de educación superior. Revista Bio Ciências, v.2, n.3, p.216-223, 2013.
SALEMDEEB, R.; VIVANCO, D. F.; AL-TABBAA, A.; KHJ, E.; ERMGASSEN, Z.. A holistic approach to the environmental evaluation of food waste prevention. Waste Management, v.59, p.442-450, 2017.

SOUTO, F. B. D.; RAIMAM, M. P.; ALBINO, U. B.. Resíduos Sólidos Urbanos em Porto de Moz - PA: Problemas e Oportunidades. Rev. Geogr. Acadêmica, Boa vista, v.7, n.2, 2013.

TEIXEIRA JÚNIOR, L. C.; SOUZA, P. R. N.; SILVA, J. A. F.; FERREIRA, M. I. P.. Avaliação da produção e gestão dos resíduos sólidos de municípios da Região dos Lagos do estado do Rio de Janeiro. Boletim do Observatório Ambiental Alberto Ribeiro Lamego, Campos dos Goytacazes, v.6, n.1, p.11-27, 2012.

THANH, N. P.; MATSUI, Y.; FUJIWARA, T.. Household solid waste generation and characteristic in a Mekong Delta city, Vietnam. Journal of Environmental Management, v.91, n.11, p.2307-2321, 2010.

WALKER, M.; THEAKER, H.; YAMAN, R.; POGGIO, D.; NIMMO, W.; BYWATER, A.; BLANCH, G.. Assessment of micro-scale anaerobic digestion for management of urban organic waste: A case study in London, UK. Waste Management, v.61, p.258-268, 2017

WILLERSINN, C.; MOURON, P.; MACK, G.; SIEGRIST, M.. Food loss reduction from an environmental, socio-economic and consumer perspective - The case of the Swiss potato market. Waste Management, v.59, p.451-464, 2017.

XIAO, L.; LIN, T.; CHEN, S.; ZHANG, G.; YE, Z.; YU, Z. Characterizing Urban Household Waste Generation and Metabolism Considering Community Stratification in a Rapid Urbanizing Area of China. PLoS ONE, v.10, n.12, p.1-16, 2015.

ZENG, C.; NIU, D.; LI, H.; ZHOU, T.; ZHAO, Y.. Public perceptions and economic values of source-separated collection of rural solid waste: A pilot study in China. Resources, Conservation and Recycling, v.107, p.166-173, 2016.

ZORPAS, A. A.; LASARIDI, K.; POCIOVALISTEANU, D. M.; LOIZA, P.. Monitoring and evaluation of prevention activities regarding household organics waste from insular communities. Journal of Cleaner Production, v.172, p.3567-3577, 2018. DOI: https://doi.org/10.1016/i.jclepro.2017.03.155

A CBPC - Companhia Brasileira de Produção Científica (CNPJ: 11.221.422/0001-03) detém os direitos materiais desta publicação. Os direitos referem-se à publicação do trabalho em qualquer parte do mundo, incluindo os direitos às renovações, expansões e disseminações da contribuição, bem como outros direitos subsidiários. Todos os trabalhos publicados eletronicamente poderão posteriormente ser publicados em coletâneas impressas sob coordenação da Sustenere Publishing, da Companhia Brasileira de Produção Científica e seus parceiros autorizados. Os (as) autores (as) preservam os direitos autorais, mas não têm permissão para a publicação da contribuição em outro meio, impresso ou digital, em português ou em tradução. 